\title{
Multi-UAV Deployment for Throughput Maximization in the Presence of Co-Channel Interference
}

\author{
Iman Valiulahi, Christos Masouros, Senior Member, IEEE
}

\begin{abstract}
Over the past few years, there has been a growing interest in using Unmanned Aerial Vehicles (UAVs) for highrate wireless communication systems due to their highly flexible deployment and maneuverability. The aim of this paper is to propose a three dimensional (3D) multi-UAV deployment approach to provide quality of service $(\mathrm{QoS})$ requirements for different types of user distributions in the presence of co-channel interference by maximizing the minimum achievable system throughput for all of the ground users. The proposed approach is divided into two separate algorithms. In the first algorithm, by using the mean-shift technique and prior knowledge of users' positions provided by Global Positioning System (GPS), it has been shown that one can simultaneously find $x y$ coordinates of UAVs where are associated with the maximum of users' density and schedule users to UAVs. Once the $x y$-Cartesian coordinates of UAVs are determined, UAVs altitudes and transmit powers are separately optimized. Since these problems are non-convex optimizations, the successive convex optimization technique has been applied to approximate their non-convex constraints. In the second algorithm, the block coordinate descent technique is leveraged to jointly optimize UAVs altitudes and transmit powers by tightening the bounds obtained for approximations. It is then proven that the suggested algorithm is guaranteed to converge. The computational complexity of the proposed placement approach is derived. Numerical experiments are carried out to evaluate the performance of our technique and show its superiority to conventional benchmarks.
\end{abstract}

Index Terms-3D deployment, unmanned aerial vehicles, mean-shift technique, altitude and transmit power optimizations.

\section{INTRODUCTION}

Unmanned aerial vehicles (UAVs) have recently attracted interest as a rapid solution for providing communication services to ground users [1], [2]. In practice, it is not cost-effective or even possible to deploy terrestrial base stations (BSs) in temporary hotspots or disaster areas. On the other hand, due to the highly flexible deployment and maneuverability of UAVs, they can be employed in an efficient manner to serve as aerial BSs [3]-[15]. Moreover, the communication link between users and UAVs has typically high probabilities of line-of-sight (LoS) air-to-ground (A2G) channels, which can mitigate signal blockage and shadowing [16]. In addition, UAVs can be used in internet of things (IoT) where the goal is to make connections among IoT devices that are typically battery-limited sensors and monitors [17]. The limited energy

The authors are with the Department of Electronic and Electrical Engineering, University College London, London WC1E 7JE, U.K. (e-mail: i.valiulahi@ucl.ac.uk; c.masouros@ucl.ac.uk; chris.masouros@ieee.org) constraints of the devices make long-distance communication services impossible. UAVs, however, can fly toward the IoT devices and collect their data and transmit them to other devices which might be located at far ranges [18]. Also, in the sustainable rural agriculture [19], there has been a growing interest in the potential of IoT to support poverty alleviation in rural areas without terrestrial BSs. Additionally, research is also being conducted on UAV based traffic monitoring and several techniques proposed to evaluate what is the most efficient way to transmit and analyze traffic data acquired from UAVs [20]. UAVs are superior to the conventional technologies such as video and radar due to their mobility and because the implementation cost of the operation is considerably lower than manned systems.

Although there exist many benefits with UAVs for communication purposes, deploying UAVs implicates a number of challenges. More precisely, in the existing cellular systems, the A2G link is only a function of users' positions as the locations of the terrestrial BSs are fixed. In the UAV-based communication systems, however, the path loss not only depends on users' positions but also on the UAVs' locations. Furthermore, in the multi-UAV case, handling the effects of co-channel interference on system throughput is more complicated than the conventional terrestrial BSs because UAVs can fly in arbitrary directions.

Generally speaking, there are two different lines of research focusing on UAV-based wireless communication platforms [3]. In the first scenario, a wireless communication transmitter is carried by UAVs that can fly between their served ground users [4], [5]. Trajectories of UAVs are needed to be designed in this case. In [6] and [7], authors investigated a single UAV and multi-UAV trajectory optimizations, respectively. Moreover, in [8], authors studied a single solar-powered UAV trajectory design to jointly adapt transmit power and allocate frequency subcarriers to ground users. In the second area of research, UAVs are considered as quasi-static BSs and the aim is to find the fixed locations of UAVs to provide wireless services to ground users $[9]-[12]$. More precisely, the 3D locations of UAVs are needed to be optimized for providing QoS requirements.

\section{A. Related Works}

In [11], a mathematical analysis is provided to evaluate the effect of the optimal altitude of a single UAV on the maximum coverage region. Using the minimum transmit power, in [13], 
a scheme is proposed to investigate how the $3 \mathrm{D}$ position of a UAV affects the maximum number of users served by the UAV. In [14], it is shown that when users require different QoSs, a multiple circle placement technique can be used to deploy a single UAV to maximize the number of covered users. In [21], a joint design of the 3D deployment of a single solar-powered UAV and the power and subcarrier allocation for maximizing the sum of data throughput is provided. In [22], a heuristic algorithm based on particle swarm optimization is suggested to find the minimum required number of UAVs to serve ground users in the urban environment. In [12], authors proposed a new polynomial-time algorithm for multiUAV placement that minimizes the number of UAVs required to provide communication services. In reference [10], an efficient approach based on circle packing theorem (CPT) is developed to deploy multi-UAV in the 3D case. Reference [15] made a step further and proposed an energy efficient simultaneous deployment method with variable radius (SDKMVR) to maximize user coverage and power efficiency.

In this work, we assume that there exist no terrestrial BSs and focus only on multi-UAV placement in a target area. This can happen, for example, in the case of a complex terrain where irregular topographies such as mountains or coastlines make impossible to deploy conventional terrestrial infrastructures. Following works [10] and [12], we suppose that prior information regarding users' positions is available by the high-accuracy global positioning system (GPS). We consider that all UAVs share the same frequency band for communicating with ground users. Each UAV can serve its scheduled users by a different cyclical time-division multiple access (TDMA) scheme regarding the number of its scheduled users.

In the next subsection, we mention the main contributions of this paper and briefly compare the results with the benchmarks.

\section{B. Main Contributions of This Paper}

- We propose a new 3D multi-UAV deployment based on convex optimization where we take co-channel interference into account. We show that the suggested optimization problem contains non-linear and integer constraints. To simultaneously take advantage of GPS information and get rid of the non-linear and integer constraints, we propose an algorithm based on the mean-shift technique to find $x y$-Cartesian coordinate of UAVs and schedule users to UAVs.

- When users are scheduled and $x y$ locations of UAVs are calculated, we separately optimize UAVs altitudes.

- Once the placement of UAVs is found, we optimize UAVs transmit powers in a separate block.

- To jointly optimize UAVs altitudes and transmit powers, an iterative algorithm based on the block coordinate descent [23] is developed.

- The overall computational complexity of the proposed placement approach is derived.

- Finally, the simulation results are done to investigate the performance of the proposed technique and reveal that our method outperforms the conventional benchmarks CPT

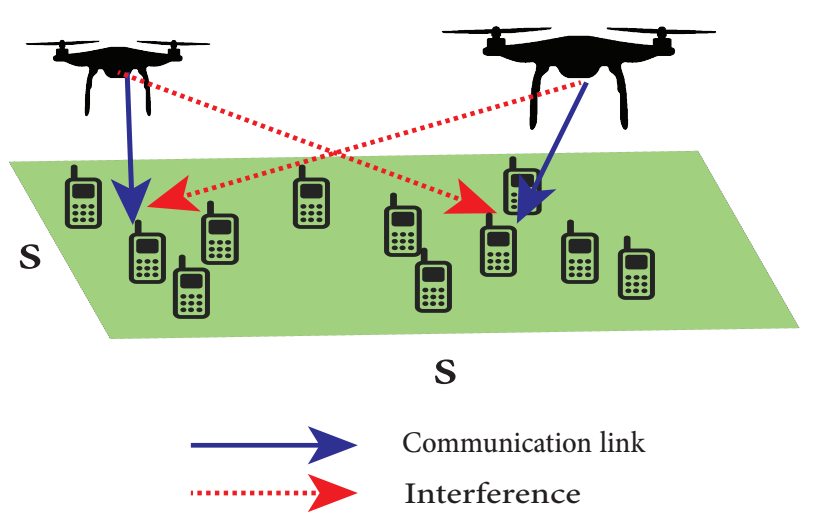

Fig. 1: System model and the target area.

and SD-KMVR in terms of system sum throughput and consumed power.

To highlight the results of this paper, we would like to summarize the following points. We maximize the minimum achievable system throughput for all ground users. Thus, the proposed optimization can obtain $100 \%$ coverage probability and provide fairness among ground users in terms of the system throughout which are significant achievements compared to the conventional methods. Moreover, three novel deploying strategies are inside of our proposed 3D multiUAVs deployment approach that outperform the benchmarks. First, one can place UAVs in the $x y$ locations where are associated with the maximum user's density via Algorithm 1. Compared to the CPT, this approach can automatically determine the number of UAVs required to serve ground users and allocate UAVs regarding the user's distribution. This leads to a significant saving in the available sources in practice. In the second deployment approach, one can deploy UAVs in the $x y$-Cartesian coordinates obtained from Algorithm 1 and optimize UAV's transmit powers. This strategy outperforms the conventional approaches, SD-KMVR and CPT. In the third approach, we show that one can jointly optimize the UAV's altitude and transmit powers in order to mitigate the effects of co-channel interference by allowing UAVs to work in different altitudes. The performance obtained in terms of system throughput for this approach is better than all other techniques.

The paper is organized as follows: the system model and achievable system throughput are given in Section II In Section III the optimization problem for multi-UAV placement is formulated. In Section IV, an algorithm based on the mean-shift technique for deploying multi-UAV in $x y$ plane is proposed. In Section V, UAVs altitudes optimization and a novel technique to implement it are presented. Section VI is devoted to UAVs transmit powers optimization. In Section VII an iterative algorithm to jointly optimize UAVs altitudes and transmit powers is developed. The overall computational cost of the proposed approach is derived in Section VIII. Numerical experiments are carried out in Section IX Finally, the paper is concluded in Section $X$

Throughout the paper, scalars are denoted by lowercase 
letters, vectors by lowercase boldface letters, and matrices by uppercase boldface letters. The $k$-th element of the vector $\boldsymbol{x}$ is shown by $x_{k}$. Function $\|\cdot\|_{2}$ is reserved for $\ell_{2}$ norm. The operators $(\cdot)^{T}$ and $E\{\cdot\}$ represent the transpose of a vector and the expectation of a random process. We use the symbol ! to show the factorial product, i.e., $n !=(n)(n-1) \cdots(2)(1)$.

\section{SySTEM MODEL}

As shown in Fig. 1, we consider a wireless communication square area with side length $s$ where $U>1$ low-mobility users are needed to be served by $M>=1$ UAVs. Assume that $\mathcal{U}=\{1, \cdots, U\}$ and $\mathcal{M}=\{1, \cdots, M\}$ are user and UAV sets, respectively. Let $\boldsymbol{u}_{u}=\left[x_{u}, y_{u}\right]^{T} \in \mathbb{R}^{2 \times 1}$ represent the horizontal coordinate of $u$-th ground user where $u \in \mathcal{U}$. We use $\boldsymbol{m}_{m}=\left[x_{m}, y_{m}, z_{m}\right]^{T} \in \mathbb{R}^{3 \times 1}$ to denote the 3D Cartesian coordinate of $m$-th UAV where $m \in \mathcal{M}$. Without loss of generality, let us assume $\overline{\boldsymbol{m}}_{m}=\left[x_{m}, y_{m}\right]^{T}$ where $\overline{\boldsymbol{m}}_{m}$ is the horizontal coordinate of $m$-th UAV and $z=\left[z_{1}, \cdots, z_{M}\right]^{T}$ where $h_{\min } \leq z_{m} \leq h_{\max }$ in which $h_{\min }$ and $h_{\max }$ are used to show the minimum and maximum allowed altitude of $m$-th UAV, respectively. We also suppose that the down-link transmit power of $m$-th UAV satisfies $p_{\text {min }} \leq p_{m} \leq p_{\max }$, where $p_{\text {min }}$ and $p_{\max }$ are associated with the minimum and maximum transmit power, respectively. To avoid collision among UAVs, the horizontal distance between $m$-th and $j$-th UAVs is subject to the following constraint

$$
\left\|\overline{\boldsymbol{m}}_{m}-\overline{\boldsymbol{m}}_{j}\right\|_{2} \geq d_{\mathrm{min}}, \quad m \text { and } j \in \mathcal{M},
$$

where $d_{\min }$ shows the minimum allowed distance among UAVs.

It is worth mentioning that this work is not involved with a trajectory design. We indeed investigate one snapshot of time and its associated users' positions. To compensate users' movements, one is able to regularly repeat the proposed approach with updated users' information.

\section{A. Achievable system throughput in the presence of co-channel interference}

In practice, the ground users receive three different kinds of signals from UAVs including LoS, non-line-of-sight (NLoS), and multiple reflected signals [24], [11]. These signals occur with specific probabilities in different environments. As discussed in [25], the probability of multiple reflected signal which causes multi-path fading is significantly lower than two other signals. Thus, the effect of this signal at the receivers is typically ignored. Here, in line with the literature [7], [8], we assume that the communication link between ground users and UAVs is dominated by the LoS signals. Under this assumption, the channel power gain between $u$-th users and $m$-th UAV is only a function of their Euclidean distance as below

$$
h_{u, m}=\rho_{0} d_{u, m}^{-2},
$$

where $\rho_{0}$ is a constant showing the power of the channel at the reference distance $d_{0}=1 \mathrm{~m}$ and $d_{u, m}$ is the Euclidean distance between $u$-th user and $m$-th UAV which can be written as

$$
d_{u, m}=\sqrt{z_{m}^{2}+\left\|\boldsymbol{u}_{u}-\overline{\boldsymbol{m}}_{m}\right\|_{2}^{2}} .
$$

Hence, we have

$$
h_{u, m}=\frac{\rho_{0}}{z_{m}^{2}+\left\|\boldsymbol{u}_{u}-\overline{\boldsymbol{m}}_{m}\right\|_{2}^{2}} .
$$

As mentioned, the aim is to deploy multi-UAV, thus, each ground user needs to be associated only by one UAV. Let define a binary variable $\alpha_{u, m}$ for $u$-th user and $m$-th UAV. If $u$-th user is scheduled by $m$-th UAV, $\alpha_{u, m}=1$, otherwise, $\alpha_{u, m}=0$. To satisfy these conditions, one can use the following constraints

$$
\begin{aligned}
& \sum_{m=1}^{M} \alpha_{u, m} \leq 1, \\
& \alpha_{u, m} \in\{0,1\}, \quad u \in \mathcal{U}, \text { and } m \in \mathcal{M} .
\end{aligned}
$$

However, in Section IV we show that we can circumvent the above linear and integer constraints using the mean-shift technique.

Regarding the transmit power and user association, the achievable system throughput in bits/second/Hertz (bit/s/Hz) for $u$-th user can be formulated as below

$$
R_{u}=\sum_{m=1}^{M} \alpha_{u, m} \log _{2}\left(1+\gamma_{u, m}\right), \quad u \in \mathcal{U},
$$

where $\gamma_{u, m}$ is the signal-to-interference-plus-noise ratio (SINR) corresponding to the $u$-th user and $m$-th UAV, which can be expressed as

$$
\gamma_{u, m}=\frac{p_{m} h_{u, m}}{\sum_{r=1, r \neq m}^{M} p_{r} h_{u, r}+\sigma^{2}}, \quad u \in \mathcal{U}, \text { and } m \in \mathcal{M}
$$

where $\sigma^{2}$ is the power of the additive white Gaussian noise (AWGN) at $u$-th user. Note that due to the TDMA operation, there is no interference from the users served by the same UAV, and the term $\sum_{r=1, r \neq m}^{M} p_{r} h_{u, r}$ is caused because of cochannel interference of other UAVs at $u$-th user. Consequently, $R_{u}$ can be recast as below

$$
R_{u}=\sum_{m=1}^{M} \alpha_{u, m} \log _{2}\left(1+\frac{p_{m} h_{u, m}}{\sum_{r=1, r \neq m}^{M} p_{r} h_{u, r}+\sigma^{2}}\right),
$$

for $u \in \mathcal{U}$.

As a result of the multi-UAV placement, co-channel interference could be large enough to obstruct the communication links between UAVs and their scheduled users. Accordingly, specifically tailored power allocation is needed to mitigate the effects of co-channel interference. Moreover, using the GPS, users' positions are known with high accuracy in advance. It will be beneficial if one incorporates this additional information into the placement strategy while considering co-channel interference. In the next section, we formalize an optimization problem that considers these features.

\section{PROBLEM FORMULATION}

In this section, we propose an optimization problem that allocates communication services to all ground users and hence achieves $100 \%$ coverage probability, while considering all aforementioned conditions for deploying. Let first define 


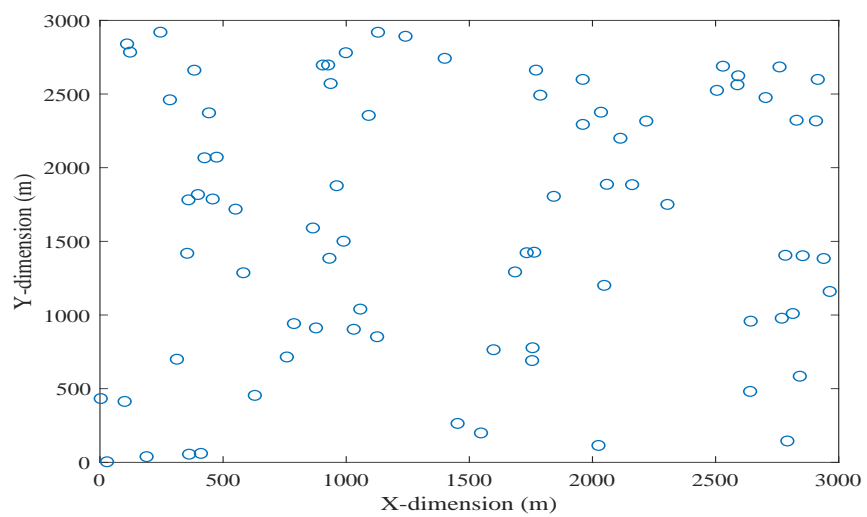

(a)

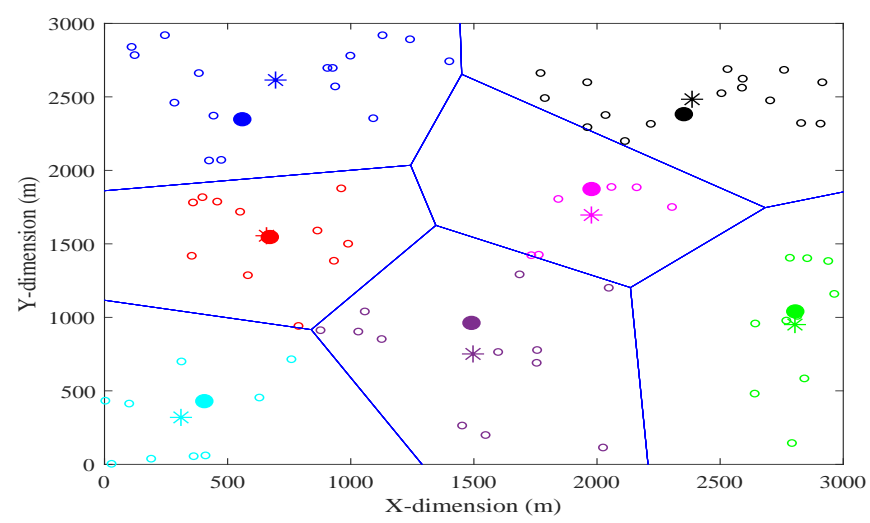

(b)

Fig. 2: Fig. 2(a) demonstrates users' positions in blue circles. Fig. 2(b) shows user classification using Algorithm 1 Colored dots are used to denote the mean-shift centers. Voronoi diagram constructed by the mean-shift centers is drawn in blue lines. The associated users with each center are colored by the same color as the center points. The means of scheduled users within each convex region are shown in the colored stars.

$\boldsymbol{p}:=\left[p_{1}, \cdots, p_{M}\right]^{T}$ the transmit power vector, $\boldsymbol{L} \in \mathbb{R}^{3 \times M}$ as the position matrix that its $m$-column is corresponded to the 3D Cartesian coordinate of $m-\mathrm{UAV}$, and $\boldsymbol{A} \in \mathbb{R}^{U \times M}$ as the scheduling matrix that its element is associated with $\alpha_{u, m}$. To provide communication services for all ground users, we maximize the minimum of the achievable system throughput of each user subject to mentioned placement conditions as below

$$
\begin{aligned}
& \max _{\psi_{c}, \boldsymbol{p}, \boldsymbol{A}, \boldsymbol{L}} \psi_{c} \\
& \text { s.t. } \sum_{m=1}^{M} \alpha_{u, m} \log _{2}\left(1+\frac{p_{m} h_{u, m}}{\sum_{r=1, r \neq m}^{M} p_{r} h_{u, r}+\sigma^{2}}\right) \geq \psi_{c} \\
& u \in \mathcal{U} \\
& \sum_{m=1}^{M} \alpha_{u, m} \leq 1 \\
& \alpha_{u, m} \in\{0,1\}, \quad u \in \mathcal{U} \text { and } m \in \mathcal{M} \\
& p_{\min } \leq p_{m} \leq p_{\max }, \quad m \in \mathcal{M} \\
& h_{\min } \leq z_{m} \leq h_{\max }, \quad m \in \mathcal{M} \\
& \left\|\overline{\boldsymbol{m}}_{m}-\overline{\boldsymbol{m}}_{j}\right\|_{2} \geq d_{\min }, \quad m \text { and } j \in \mathcal{M} .
\end{aligned}
$$

where $\psi_{c}$ is an optimization constant variable. Constraint (9a) provides a situation in which all users access the minimum QoS requirements. On the other hand, maximizing $\psi_{c}$, it is a guarantee to obtain the maximum of this minimum service. Indeed, this is a max-min optimization. Constraint (9b) stands for user scheduling and $(9 \mathrm{c})$ restricts the UAVs transmit powers. Constraint (9d) is because of the minimum and maximum allowed altitudes. Finally, constraint $9 \mathrm{e}$ ensures collision avoidance.

Solving (9) is difficult due to non-convex constraints 9a and $(9 \mathrm{e})$ as well as integer constraints $(9 \mathrm{~b})$. Moreover, this is a non-convex optimization over a $3 \mathrm{D}$ variable $\boldsymbol{L}$, which is generally an obstacle to handle in the presence of cochannel interference. The original optimization problem in (9) is divided into four stages. More precisely, we first propose a low complex algorithm based on the mean-shift technique to

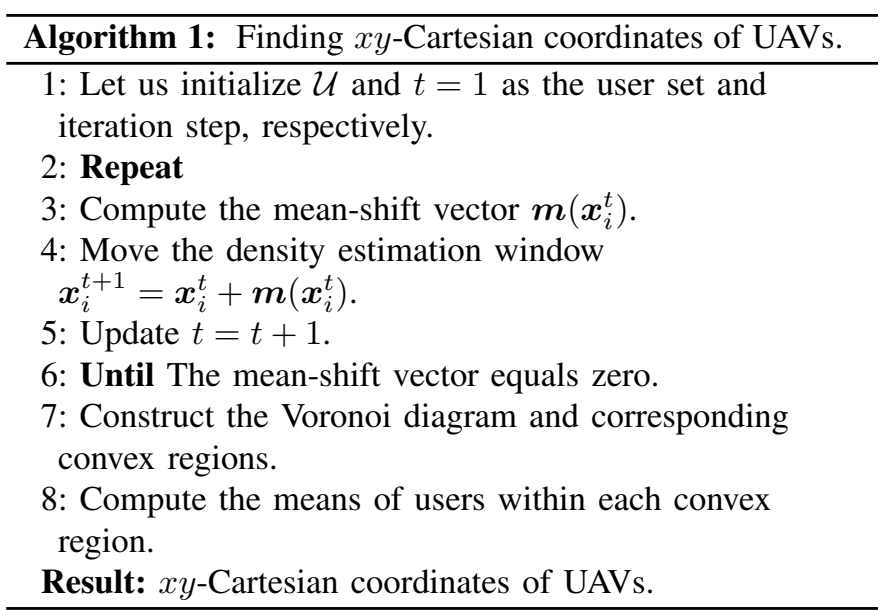

obtain the $x y$-Cartesian coordinate of UAVs and schedule the ground users to UAVs. Then, we separately optimize UAVs altitudes and transmit powers in the second and third stages, respectively. In the last stage, we develop an iterative algorithm based on the block coordinate descent to jointly optimize UAVs altitudes and transmit powers. In the next sections, we present these stages to overcome the difficulties of problem (9).

\section{Stage 1: Finding $x y$-Cartesian Coordinates of UAVS}

As discussed, problem 9 is a combination of non-convex and integer constraints over the $3 \mathrm{D}$ variable. Thus, it is challenging to solve. To simplify this problem, we take advantage of prior information regarding users' positions to divide the target area to $M$ non-overlap partitions using the mean-shift technique. This can schedule users to UAVs and get rid of collision avoidance.

The mean-shift technique is a non-parametric approach to find the maximum density of a function. Applications for the mean-shift are vast from computer vision to image processing [26]. However, to the best of our knowledge, this paper is the 
first work that uses the mean-shift technique to classify ground users in order to deploy multi-UAV. As opposed to alternative clustering approaches such as K-means [15], the beauty of the mean-shift is that the number of clusters is not required to be known in advance. Indeed, the mean-shift technique can automatically extract the number of clusters. In our work, this means that the number of UAVs required ro serve ground users is determined by our prior information regarding users' positions, which is very useful in practice.

To exploit the mean-shift technique, let us consider $\boldsymbol{x}_{i} \in \mathbb{R}^{2}$ for $i \in\{1, \cdots, U\}$ as an arbitrary set of users in the 2D Euclidean space (see Fig. 2 (a)). Let us use Epanechnikov kernel that can achieve the minimum mean integrated error (MISE) [26] as below

$$
K(\boldsymbol{x})=\left\{\begin{array}{lr}
\frac{2}{c_{d}}\left(1-\|\boldsymbol{x}\|_{2}^{2}\right) & \text { if }\|\boldsymbol{x}\|_{2}^{2} \leq 1, \\
0 & \text { otherwise, }
\end{array}\right.
$$

where $c_{d}$ is the area of the unit circle. The multivariate kernel density estimate with $\lambda$-radius using the above kernel can be written as below

$$
\hat{f}(\boldsymbol{x})=\frac{1}{U \lambda^{2}} \sum_{i=1}^{U} K\left(\frac{\boldsymbol{x}-\boldsymbol{x}_{i}}{\lambda}\right) .
$$

Defining $g(\boldsymbol{x}):=-K^{\prime}(\boldsymbol{x})$, we can write the mean-shift vector as

$$
\boldsymbol{m}(\boldsymbol{x})=\frac{\sum_{i=1}^{U} g\left(\frac{\boldsymbol{x}-\boldsymbol{x}_{i}}{\lambda}\right) \boldsymbol{x}_{i}}{\sum_{i=1}^{U} g\left(\frac{\boldsymbol{x}-\boldsymbol{x}_{i}}{\lambda}\right)}-\boldsymbol{x} .
$$

Let us denote the iteration index of the mean-shift technique as $t$. The mean-shift technique can be summarized as computing the mean-shift vector $\boldsymbol{m}\left(\boldsymbol{x}_{i}^{t}\right)$, moving the density estimation window $\boldsymbol{x}_{i}^{t+1}=\boldsymbol{x}_{i}^{t}+\boldsymbol{m}\left(\boldsymbol{x}_{i}^{t}\right)$ until the mean-shift vector equals zero. Assume that $\left\{y_{k}\right\}_{k=1,2, \cdots}$ is the sequence of the positions of the mean-shift procedure. By setting $\lambda=d_{\min } / 2$ and the results provided in [26], it is possible to prove that the density sequence $\hat{f}_{E}=\left\{\hat{f}\left(y_{k}\right), K\right\}$ computed at the points $\left\{y_{k}\right\}_{k=1,2, \ldots}$ using Epanechnikov kernel, $K$ in 10 , is convergent for any data distribution. In our work, it means that the mean-shift technique can classify users no matter how they are distributed. Another important point is that one can control the area around each mean-shift center by changing $d_{\text {min }}$. This comes from the fact that $\lambda$, the radius of the multivariate kernel, depends on $d_{\text {min }}$. Indeed, by increasing $d_{\min }, \lambda$ increases and the number of mean-shift centers to classify the user distribution decreases and vice versa.

Once the mean-shift centers are extracted (see colored dots in Fig. 2 (b)), we construct the Voronoi diagram using these points. We then build the convex hull corresponding to Voronoi points. Users that are associated with each convex region can be determined (colored circles in Fig. 2(b)). We then calculate the mean of users' locations within each convex hull where are associated with the maximum density of users in that convex region (colored stars in Fig. 2 (b)). Now, we consider these locations as the $x y$-Cartesian coordinate of UAVs. For ease of notations, in the sequel, we use $\hat{\boldsymbol{m}}_{m}=\left[x_{m}, y_{m}\right]^{T}$ as the $x y$ position of $m$-th UAV and $\hat{\alpha}_{u, m}$ as the binary user scheduling, showing that $u$-th user is served by $m$-th UAV. The details of the proposed algorithm are summarized in Algorithm 1 .
There are three major benefits to this classification. First, we schedule ground users to UAVs. Thus, the binary constraint in (9b) is eliminated from the optimization in (9) as Algorithm 1 determined which users must be served by which UAV. Second, the $x y$-Cartesian coordinates of UAVs that are associated with the maximum density of users in each convex region are calculated, thus the altitudes of UAVs are the only unknown variables for UAVs' positions. Third, non-convex constraint (9e) for collision avoidance is satisfied by setting $\lambda=d_{\min } / 2$ in (11).

Therefore, by defining $\psi(\boldsymbol{z}, \boldsymbol{p}):=\min _{u \in \mathcal{U}} R_{u}$ as a function of $\boldsymbol{z}$ and $\boldsymbol{p}$, we recast problem (9) as below

$$
\begin{aligned}
& \max _{\psi, \boldsymbol{z}, \boldsymbol{p}} \quad \psi \\
& \text { s.t. } \hat{\alpha}_{u, m} \log _{2}\left(1+\frac{p_{m} h_{u, m}}{\sum_{r=1, r \neq m}^{M} p_{r} h_{u, r}+\sigma^{2}}\right) \geq \psi, u \in \mathcal{U} \\
& p_{\min } \leq p_{m} \leq p_{\max }, \quad m \in \mathcal{M} \\
& h_{\min } \leq z_{m} \leq h_{\max }, \quad m \in \mathcal{M} .
\end{aligned}
$$

Note that the summation in constraint $9 \mathrm{a}$ is removed because users are scheduled to UAVs by Algorithm 1

Despite the above simplifications, it is still hard to implement problem 13 because of non-convex constraint 13a respect to $\boldsymbol{z}$ and $\boldsymbol{p}$. In Section $\mathrm{V}$, we first optimize UAVs altitudes for given transmit power $\boldsymbol{p}$. Then, in Section VI we optimize transmit power for given UAVs altitudes $z$. Based on the fact that these two optimizations are not convex, we take advantage of the successive convex optimization technique to relax them and make them convex. In Section VII. we then propose an iterative algorithm based on the block coordinate descent to jointly optimize UAVs altitudes and transmit powers.

Note that Algorithm 1 can classify any user distribution, thus, the number of ground users served by each UAV might be different. To avoid unfairness for the users in a cluster with large number of users, we propose TDMA schemes with different time slots. More precisely, assuming that UAVs are required to serve their scheduled users during the transmission time $[0, T)$ after deploying and the number of users served by each UAV is $\tilde{U}_{m}$ for $m \in \mathcal{M}$. By defining $\bar{t}_{m}:=\frac{T}{\tilde{U}_{m}}$ for $m \in \mathcal{M}$ as the time slot associated with each UAV, we are able to provide fairness among the users. Indeed, this strategy ensures that all users access to communication services during the transmission interval.

\section{Stage 2: UAVs Altitudes Optimization}

Generally speaking, it is tough to simultaneously optimize $\boldsymbol{z}$ and $p$ in problem (13) because constraint $(13 \mathrm{a})$ is non-convex respect to both of these variables. To tackle this issue, we first try to optimize UAVs altitudes for given transmit power $\boldsymbol{p}$. To 
do so, we recast problem (13) as below

$$
\begin{aligned}
& \max _{\psi, \boldsymbol{z}} \psi \\
& \text { s.t. } \hat{\alpha}_{u, m} \log _{2}\left(1+\frac{\frac{p_{m} \rho_{0}}{z_{m}^{2}+\left\|\hat{\boldsymbol{m}}_{m}-\boldsymbol{u}_{u}\right\|_{2}^{2}}}{\sum_{r=1, r \neq m}^{M} \frac{p_{r} \rho_{0}}{z_{r}^{2}+\left\|\hat{\boldsymbol{m}}_{r}-\boldsymbol{u}_{u}\right\|_{2}^{2}}+\sigma^{2}}\right) \geq \psi \\
& u \in \mathcal{U} \\
& h_{\min } \leq z_{m} \leq h_{\max }, \quad m \in \mathcal{M} .
\end{aligned}
$$

Note that $\hat{\alpha}$ and $\hat{\boldsymbol{m}}$ are obtained after applying Algorithm 1. There exists no efficient approach to solve the above optimization problem because constraint $[14 \mathrm{a})$ is not convex respect to $z$. To handle this situation, let us first rewrite the logarithm in 14a as follows

$$
\begin{aligned}
\log _{2}\left(1+\frac{\frac{p_{m} \rho_{0}}{z_{m}^{2}+\left\|\hat{\boldsymbol{m}}_{m}-\boldsymbol{u}_{u}\right\|_{2}^{2}}}{\sum_{r=1, r \neq m}^{M} \frac{p_{r} \rho_{0}}{z_{r}^{2}+\left\|\hat{\boldsymbol{m}}_{r}-\boldsymbol{u}_{u}\right\|_{2}^{2}}+\sigma^{2}}\right) \\
\quad=G(\boldsymbol{z})-\log _{2}\left(\sum_{r=1, r \neq m}^{M} \frac{p_{r} \rho_{0}}{z_{r}^{2}+\left\|\hat{\boldsymbol{m}}_{r}-\boldsymbol{u}_{u}\right\|_{2}^{2}}+\sigma^{2}\right),
\end{aligned}
$$

where

$$
G(\boldsymbol{z}):=\log _{2}\left(\sum_{r=1}^{M} \frac{p_{r} \rho_{0}}{z_{r}^{2}+\left\|\hat{\boldsymbol{m}}_{r}-\boldsymbol{u}_{u}\right\|_{2}^{2}}+\sigma^{2}\right)
$$

for any $u \in \mathcal{U}$.

It is worth noting that equation (15) is a difference of two convex functions respect to $z$. Thus, it is still non-convex. Regarding the fact that there exists a globally lower bound on an arbitrary convex function, $f(\boldsymbol{t})$, at an arbitrary point, $\tilde{\boldsymbol{t}}$, using its first-order Taylor expansion as below [27]

$$
f(\boldsymbol{t}) \geq f(\tilde{\boldsymbol{t}})+\nabla_{\boldsymbol{t}} f(\tilde{\boldsymbol{t}})^{T}(\boldsymbol{t}-\tilde{\boldsymbol{t}})
$$

the lower bound on $G(\boldsymbol{z})$ at the local points $\tilde{\boldsymbol{z}}=$ $\left[\tilde{z}_{1}^{2}, \cdots, \tilde{z}_{M}^{2}\right]^{T}$ is given by

$$
\begin{aligned}
\log _{2}( & \left.\sum_{r=1}^{M} \frac{p_{r} \rho_{0}}{z_{r}^{2}+\left\|\hat{\boldsymbol{m}}_{r}-\boldsymbol{u}_{u}\right\|_{2}^{2}}+\sigma^{2}\right) \\
\geq & \log _{2}\left(\sum_{r=1}^{M} \frac{p_{r} \rho_{0}}{\tilde{z}_{r}^{2}+\left\|\hat{\boldsymbol{m}}_{r}-\boldsymbol{u}_{u}\right\|_{2}^{2}}+\sigma^{2}\right) \\
& +\sum_{r=1}^{M} w_{\tilde{\boldsymbol{z}}}(u, r)\left(z_{r}^{2}-\tilde{z}_{r}^{2}\right):=\bar{G}(\boldsymbol{z}),
\end{aligned}
$$

where

$$
w_{\tilde{\boldsymbol{z}}}(u, r):=\frac{-\frac{p_{r} \rho_{0}}{\left(\tilde{z}_{r}^{2}+\left\|\hat{\boldsymbol{m}}_{r}-\boldsymbol{u}_{u}\right\|_{2}^{2}\right)^{2} \ln 2}}{\sum_{l=1}^{M} \frac{p_{l} \rho_{0}}{\tilde{z}_{l}^{2}+\left\|\hat{\boldsymbol{m}}_{l}-\boldsymbol{u}_{u}\right\|_{2}^{2}}+\sigma^{2}} .
$$

Note that the derivative is taken respect to $z^{2}$. By defining variables $v_{m}:=z_{m}^{2}$ for $m \in \mathcal{M}$ in 15 and the above bound, problem 14 can be recast as

$\max _{\psi_{z}, \boldsymbol{z}, \boldsymbol{v}} \psi_{z}$

s.t.

$\hat{\alpha}_{u, m}(\bar{G}(\boldsymbol{z})$

$\left.-\log _{2}\left(\sum_{r=1, r \neq m}^{M} \frac{p_{r} \rho_{0}}{v_{r}+\left\|\hat{\boldsymbol{m}}_{r}-\boldsymbol{u}_{u}\right\|_{2}^{2}}+\sigma^{2}\right)\right) \geq \psi_{z}, \quad u \in \mathcal{U}$,

$v_{r} \leq z_{r}^{2} \quad \forall u, r \neq m$,

$h_{\min } \leq z_{m} \leq h_{\max }, \quad m \in \mathcal{M}$,

where $\psi_{z}:=\psi_{\boldsymbol{z}}(\boldsymbol{z}, \boldsymbol{p})$. Without loss of optimality of this problem, it can be verified that all constraints in 19b hold with equality, otherwise we can always increase $v$ without decreasing the objective value. Moreover, with respect to the fact that the set generated by constraint $19 \mathrm{~b}$ is the superlevel set of a convex function, it is a non-convex set [27]. To handle this situation, we again leverage the first-order Taylor expansion technique for $z^{2}$ at the local point $\tilde{z}$ as below

$$
z^{2} \geq \tilde{z}^{2}+2 \tilde{z}(z-\tilde{z})
$$

Then, we have

$\max _{\psi_{z, \boldsymbol{z}, \boldsymbol{v}}} \psi_{z}$

s.t.

$\hat{\alpha}_{u, m}(\bar{G}(\boldsymbol{z})$

$\left.-\log _{2}\left(\sum_{r=1, r \neq m}^{M} \frac{p_{r} \rho_{0}}{v_{r}+\left\|\hat{\boldsymbol{m}}_{r}-\boldsymbol{u}_{u}\right\|_{2}^{2}}+\sigma^{2}\right)\right) \geq \psi_{z}, \quad u \in \mathcal{U}$,

$v_{r} \leq \tilde{z}_{r}^{2}+2 \tilde{z}_{r}\left(z_{r}-\tilde{z}_{r}\right) \quad \forall u, r \neq m$,

$h_{\text {min }} \leq z_{m} \leq h_{\max }, \quad m \in \mathcal{M}$.

The lower bound obtained by the first-order Taylor expansion in (17) makes the feasible set of problem (21) serves as a subset for the feasible set of problem (14). Thus, the optimal objective value in problem (21) is always less than the optimal objective value in problem (14).

\section{A. UAVs Altitudes Implementation}

One is not able to implement problem 21) by the off-theshelf convex solvers in the multi-UAV case though its constraints are convex. In the following, we present an equivalent convex problem using a combination of exponential functions, which can be implemented by convex solvers. More precisely, the implementation issue of problem (21) is that variable $v$ is needed to be optimized inside of the logarithm. The simple case $\log _{2}\left(\frac{1}{x}+\sigma^{2}\right)$ can not indeed be implemented by the 
available CVX functions 1 as a convex solver. In the 2 UAVs system, however, one can use the following trick

$$
\begin{aligned}
\log _{2}\left(\frac{1}{x}+\sigma^{2}\right) & =\log _{2}\left(\frac{1+\sigma^{2} x}{x}\right) \\
& =-\log _{2}\left(\frac{x}{1+\sigma^{2} x}\right) \\
& =-\log _{2}\left(\frac{1}{\sigma^{2}}\left(\frac{\sigma^{2} x+1-1}{1+\sigma^{2} x}\right)\right) \\
& =-\log _{2}\left(\frac{1}{\sigma^{2}}\left(1-\frac{1}{1+\sigma^{2} x}\right)\right)
\end{aligned}
$$

where the argument of the logarithm can be implemented by a concave function. But, this technique can not be applied for the multi-UAV scenario because of the summation inside of the logarithm in 21a). Therefore, we use the following technique based on a combination of exponential functions to convert problem (21) to an implementable convex optimization. Due to the fact that the logarithm is an increasing function, the following constraints

$$
\log _{2}\left(\frac{1}{x_{1}}+\cdots+\frac{1}{x_{n}}\right) \leq t, \quad x_{i}>0
$$

can be replaced by

$$
\begin{aligned}
& \log _{2}\left(\mathrm{e}^{y_{1}}+\cdots+\mathrm{e}^{y_{n}}\right) \leq t, \\
& x_{i} \geq \mathrm{e}^{-y_{i}}, \quad i \in\{1, \cdots, n\} .
\end{aligned}
$$

Then, problem (21) can be given by

$$
\begin{aligned}
& \max _{\substack{\boldsymbol{z}, \boldsymbol{z}, \boldsymbol{v}, \boldsymbol{y} \\
\text { s.t. }}} \psi_{z} \\
& \hat{\alpha}_{u, m}(G(\boldsymbol{z}) \\
& \left.\quad-\log _{2}\left(\sum_{r=1, r \neq m} \mathrm{e}^{y_{r}+\ln \left(p_{r} \rho_{0}\right)}+\sigma^{2}\right)\right) \geq \psi_{z}, \quad u \in \mathcal{U}
\end{aligned}
$$$$
v_{r}+\left\|\hat{\boldsymbol{m}}_{r}-\boldsymbol{u}_{r}\right\|_{2}^{2}+\sigma^{2} \geq \mathrm{e}^{-y_{r}}, \quad u \in \mathcal{U}, r \neq m,
$$

$v_{r} \leq \tilde{z}_{r}^{2}+2 \tilde{z}_{r}\left(z_{r}-\tilde{z}_{r}\right) \quad u \in \mathcal{U}, r \neq m$,

$h_{\min } \leq z_{m} \leq h_{\max }, \quad m \in \mathcal{M}$,

where all constraints in 24 are convex as well as implementable by off-the-shelf convex solvers such as CVX.

Note that the proposed approach paves the way for implementing not only multi-UAV deployment optimization but also the multi-UAV trajectory optimization where the $3 \mathrm{D}$ positions of UAVs are needed to be optimized in each time slot. Thus, one is able to use the presented implementation technique for this case.

\footnotetext{
${ }^{1}$ As discussed in CVX guide [28], the argument of a logarithm function must be a concave function, however, inv_pos $(x)$ to implement $\frac{1}{x}$ returns a positive convex function. Then, $\log \left(\sigma^{2}+i n v \_p o s(x)\right) / \log (2)$ for implementing $\log _{2}\left(\frac{1}{x}+\sigma^{2}\right)$ leads an error in CVX. One can see a discussion regarding this by CVX developers on http://ask.cvxr.com/t/minimize-log-1-1$\mathrm{x}$-where-0-x-inf/4039
}

\section{Stage 3: UAVs Powers (UP) Optimization}

As mentioned, the simultaneous optimization of two variables $\boldsymbol{p}$ and $\boldsymbol{z}$ is challenging. Therefore, in this section for given $\boldsymbol{z}$, we optimize the transmit power vector $\boldsymbol{p}$. Let consider the following optimization problem

$$
\begin{aligned}
& \max _{\psi, \boldsymbol{p}} \psi \\
& \text { s.t. } \hat{\alpha}_{u, m} \log _{2}\left(1+\frac{p_{m} h_{u, m}}{\sum_{r=1, r \neq m}^{M} p_{r} h_{u, r}+\sigma^{2}}\right) \geq \psi, u \in \mathcal{U}, \\
& p_{\min } \leq p_{m} \leq p_{\max }, \quad m \in \mathcal{M} .
\end{aligned}
$$

The problem is not convex because of the non-convex constraint 25a respect to $\boldsymbol{p}$. To leverage the successive convex optimization technique, let us use the same approach with the previous section by writing the logarithm function in $25 \mathrm{a}$ for $u$-th user and $m$-th UAV as below

$$
\begin{aligned}
& \log _{2}\left(1+\frac{p_{m} h_{u, m}}{\sum_{r=1, r \neq m}^{M} p_{r} h_{u, r}+\sigma^{2}}\right) \\
& \quad=\log _{2}\left(\sum_{r=1}^{M} p_{r} h_{u, r}+\sigma^{2}\right)-Q(\boldsymbol{p}),
\end{aligned}
$$

where

$$
Q(\boldsymbol{p}):=\log _{2}\left(\sum_{r=1, r \neq m}^{M} p_{r} h_{u, r}+\sigma^{2}\right) .
$$

Equation 26 is a difference of two convex functions, hence non-convex. By using (16), an upper bound on $Q(\boldsymbol{p})$ at the local point $\tilde{\boldsymbol{p}}=\left[\tilde{p}_{1}, \cdots, \tilde{p}_{M}\right]^{T}$ for $u$-th user is given by

$$
\begin{aligned}
& \log _{2}\left(\sum_{r=1, r \neq m}^{M} p_{r} h_{u, r}+\sigma^{2}\right) \\
& \quad \leq \log _{2}\left(\sum_{r=1, r \neq m}^{M} \tilde{p}_{r} h_{u, r}+\sigma^{2}\right) \\
& \quad+\sum_{r=1, r \neq m}^{M} w_{\boldsymbol{p}}^{m}(u, r)\left(p_{r}-\tilde{p}_{r}\right):=\bar{Q}(\boldsymbol{p}),
\end{aligned}
$$

where

$$
w_{\boldsymbol{p}}^{m}(u, r):=\frac{\frac{p_{r} h_{u, r}}{\ln (2)}}{\sum_{l=1, l \neq m}^{M} p_{l} h_{u, l}+\sigma^{2}} .
$$

Consequently, we can rewrite problem 25] as

$$
\begin{aligned}
& \max _{\psi_{p}, \boldsymbol{p}} \psi_{p} \\
& \text { s.t. } \\
& \hat{\alpha}_{u, m}\left(\log _{2}\left(\sum_{r=1}^{M} p_{r} h_{u, r}+\sigma^{2}\right)-\bar{Q}(\boldsymbol{p})\right) \geq \psi_{p}, u \in \mathcal{U}, \\
& p_{\min } \leq p_{m} \leq p_{\max }, \quad m \in \mathcal{M},
\end{aligned}
$$

where $\psi_{p}:=\psi_{\boldsymbol{p}}(\boldsymbol{z}, \boldsymbol{p})$ and constraint $\left.30 \mathrm{a}\right)$ is a combination of linear and convex constraints respect to $\boldsymbol{p}$. Thus, it is a convex problem, which can be efficiently solved by CVX. In 


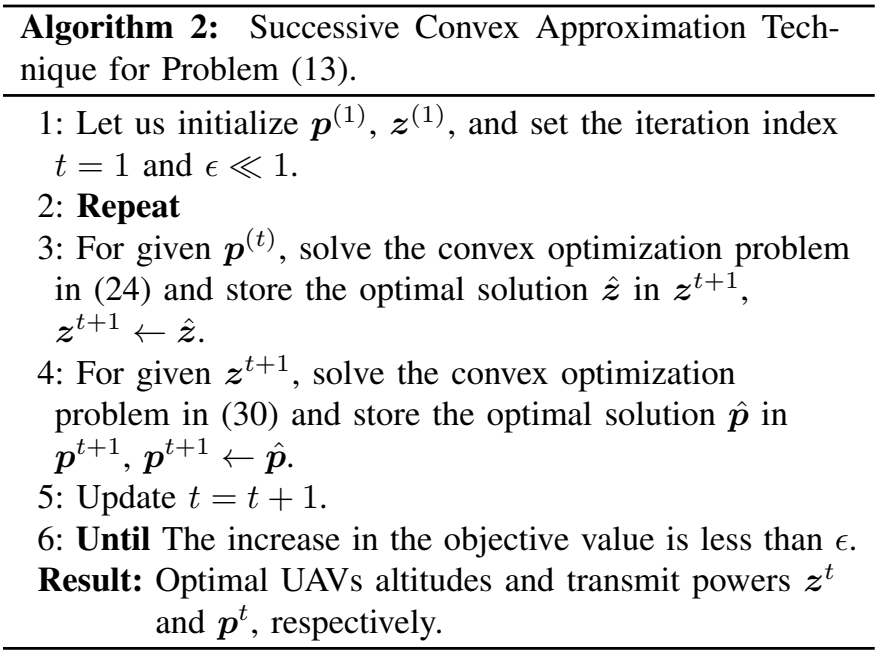

addition, the upper bound derived in (28) let the feasible set of problem (30) be a subset of the feasible set of problem (25). Accordingly, the optimal objective solution in problem 30 is always a lower bound for its counterpart in problem (25).

\section{Stage 4: JoInt UAVs Altitudes And POWERS (JUAP) OPTIMIZATIONS}

First, using Algorithm 1, users were scheduled to UAVs and the $x y$ positions of UAVs were determined. The optimization problem in (9) was then converted to problem (13). The resulted problem after user scheduling was still non-convex. The entire optimization variables, $\boldsymbol{p}$ and $\boldsymbol{z}$, were then partitioned into two separate blocks, UAVs altitudes and transmit powers optimizations in problems 24 and 30, respectively. The successive convex optimization technique was used to obtain these problems. To tighten the derived bounds for problems 24 and (30), an iterative algorithm based on the block coordinate descent approach [23] is presented in this section. The details of the proposed algorithm are summarized in Algorithm 2. After the initializing step in Algorithm 2 problem (24) is implemented to obtain $(t+1)$-th UAVs altitudes for given transmit power $\boldsymbol{p}^{(t)}$, where $t$ is the iteration step. Problem (30) is then solved in the next step to achieve $\boldsymbol{p}^{(t+1)}$ for given the altitudes solution, $\boldsymbol{z}^{t+1}$, in the previous step. Algorithm 2 is stopped when the fractional increase in the objective value is less than $\epsilon$.

In the traditional block coordinate descent algorithm, the optimal solution of each iteration is used for the next steps [23]. Here, however, the approximation of the optimal solution is obtained by the successive convex approximation technique. Thus, the convergence guarantee of Algorithm 2 is needed to be proved.

Theorem 1: If the objective value of problem [13] is upper bounded by a finite value, Algorithm 2 is convergent.

The proof of Theorem 1 is given in Appendix $\mathrm{A}$ Besides Theorem 1, in Section IX we numerically show that proposed Algorithm 2 quickly converges.

\section{Computational Complexity ANALYSIS}

In this section, we evaluate the computational complexity of the proposed deployment approach. We first analyze the complexity of finding the $x y$-Cartesian coordinates of UAVs in Algorithm 1 To compute the mean-shift vector in the third step of Algorithm 1, $U$ multiplications, $2 U$ summations, one division and one subtraction are needed, hence the computational complexity per user is $\mathcal{O}(3 U+2)$. In the next step, $U$ summations with the computational complexity $\mathcal{O}(U)$ should be implemented for each user. Let us denote the average number of iterations until convergence of the mean-shift technique as $t_{1}$. Thus, for all users $\mathcal{O}\left(t_{1}\left(3 U^{2}+U^{2}+2 U\right)\right)$ computations are needed until convergence. As explained in Algorithm 1], once the mean-shift centers are determined, we construct the Voronoi diagram with $\frac{M !}{2 !(M-2) !}=\frac{M(M-1)}{2}$ comparisons which is the combination of 2 of $M$. We then calculate the means of users in each convex region that needs $U$ summations and $M$ divisions. Consequently, the overall costs of Algorithm 1 can be approximated by

$$
\begin{aligned}
\mathcal{O}\left(t_{1}\left(4 U^{2}+2 U\right)+\frac{M(M-1)}{2}\right. & +U+M) \\
& \approx \mathcal{O}\left(t_{1}\left(4 U^{2}\right)\right),
\end{aligned}
$$

for $U \gg\{M, 1\}$ which typically satisfies in practice.

To investigate the computational complexity of Algorithm 2. note that for solving convex optimizations, CVX uses the interior point method with Newton steps [29], thus the implementation complexity of solving convex problems is

$$
\mathcal{O}\left((E+F)^{1.5} E^{2}\right)
$$

where $E$ and $F$ are the numbers of variables and constraints in the optimization problem, respectively. UAVs altitudes optimization in 24 contains $3 M+1$ variables and $3 U+M$ constraints, so the complexity is $\mathcal{O}\left((3 U+4 M+1)^{1.5}(3 M+\right.$ $\left.1)^{2}\right)$. For UAVs transmit powers optimization in $30, M+1$ variables and $U+M$ constraints are necessary to implement, hence the complexity is $\mathcal{O}\left((U+2 M+1)^{1.5}(M+1)^{2}\right)$. Let us denote $t_{2}$ as the average number of iteration until convergence of Algorithm 2. The total implementation costs of Algorithm 2 can be written as

$$
\begin{aligned}
& \mathcal{O}\left(t _ { 2 } \left((3 U+4 M+1)^{1.5}(3 M+1)^{2}\right.\right. \\
& \left.\left.\quad+(U+2 M+1)^{1.5}(M+1)^{2}\right)\right) \approx \mathcal{O}\left(t_{2}\left(48 U^{1.5} M^{2}\right)\right),
\end{aligned}
$$

for $U \gg\{M, 1\}$. Therefore, one is able to write the total computational complexity of the proposed deployment approach as

$$
\mathcal{O}\left(t_{1}\left(4 U^{2}\right)\right)+\mathcal{O}\left(t_{2}\left(48 U^{1.5} M^{2}\right)\right),
$$

which can be implemented with a moderate number of users in practice. Also, from (33), it can be inferred that the complexity of Algorithm 2 is the dominant term. The simulation experiments in Section IX supports this.

\section{NUMERICAL RESULTS}

In this section, we evaluate the performance of the proposed deployment approach by several MATLAB simulations. In the experiments, a $2 \mathrm{D}$ area of $3 \times 3 \mathrm{~km}^{2}$ is considered. 
Three different types of user distributions are studied. In this paper, a spatial point process (SPP) is used to model random user distributions. In practice, users generally follow three kinds of distributions including homogeneous Poisson process (HPP), inhomogeneous Poisson process (IPP), and Poisson cluster process (PCP) [30], [31]. For HPP and IPP, $\lambda_{s}=5$ users $/ \mathrm{km}^{2}$ and $\lambda_{s}=5\left(x^{2}+y^{2}\right)$ users $/ \mathrm{km}^{2}$ are set, respectively. Moreover, for PCP, the parent points follow HPP with the parameter $\lambda_{s}=1$ users $/ \mathrm{km}^{2}$ and the children points are generated by

$$
\lambda_{s}=\frac{\alpha_{s}}{2 \pi \sigma_{s}^{2}} \exp ^{-\frac{1}{2 \sigma_{s}^{2}}\left(x^{2}+y^{2}\right)} \text { users } / \mathrm{km}^{2},
$$

where $\alpha_{s}=0.9$ and $\sigma_{s}=0.02$. It is assumed that the minimum and maximum allowed UAVs altitudes are respectively 50 and $200 m$, i.e., $50 \leq z_{m} \leq 200$ for $m \in \mathcal{M}$. The channel power gain at the reference distance $d_{0}=1 \mathrm{~m}$ is set as $\rho_{0}=-60 \mathrm{~dB}$. The minimum and maximum allowed UAVs transmit powers are set 0.1 and $1 \mathrm{~W}$, respectively, i.e., $0.1 \leq p_{m} \leq 1$ for $m \in \mathcal{M}$. The power of dense noise is assumed to be $\sigma^{2}=-110 \mathrm{~dB}$. The UAVs altitudes and transmit powers in Algorithm 2 are initialized by the minimum and maximum allowed altitude and power, respectively, i.e., $z_{m}=50 \mathrm{~m}$ and $p_{m}=1 \mathrm{~W}$ for $m \in \mathcal{M}$. In Algorithm 2 . the parameter $\epsilon$ is set as $10^{-4}$. We use the same idea of CPT to choose $d_{\min }$ for different target areas. Regarding the fact that CPT deploys UAVs in the fixed locations, the minimum allowed distance among UAVs can be calculated for any target areas. For example, in [10], 9 UAVs are deployed to cover the target area $3 \times 3 \mathrm{~km}^{2}$. Consequently, the minimum allowed distance among UAVs is $1000 \mathrm{~m}$.

It worth pointing out that we do not study the special case with a single UAV. As there exists no co-channel interference, the maximum transmit power and the minimum altitude are optimal. The only thing is to find $x y$-Cartesian coordinate of the UAV, which can be determined by the mean-shift technique.

To evaluate the benefit of the proposed deployment approach, numerical results based on Monte Carlo simulations are carried out and the performance of our technique is compared with CPT [10] and SD-KMVR [15] as the conventional deployment methods. Note that in CPT, we deploy UAVs in the fixed locations with the minimum allowed altitude and the maximum transmit power. Also, we consider two different scenarios for our deployment approach. First, we deploy UAVs in the $x y$-Cartesian coordinates obtained by Algorithm 1 with the minimum allowed altitudes and only optimize UAVs power (UP) by removing the third step of Algorithm 2 In the second scenario, joint UAVs altitudes and powers (JUAP) optimization is considered.

\section{A. 3D Deployment}

Intuitively, there exist some trade-offs in the optimal UAVs altitudes and transmit powers in the presence of co-channel interference. More precisely, when a UAV works with the maximum power, although its associated users enjoy better communication services, it produces more interference to users served by other UAVs. Thus, the maximum transmit power

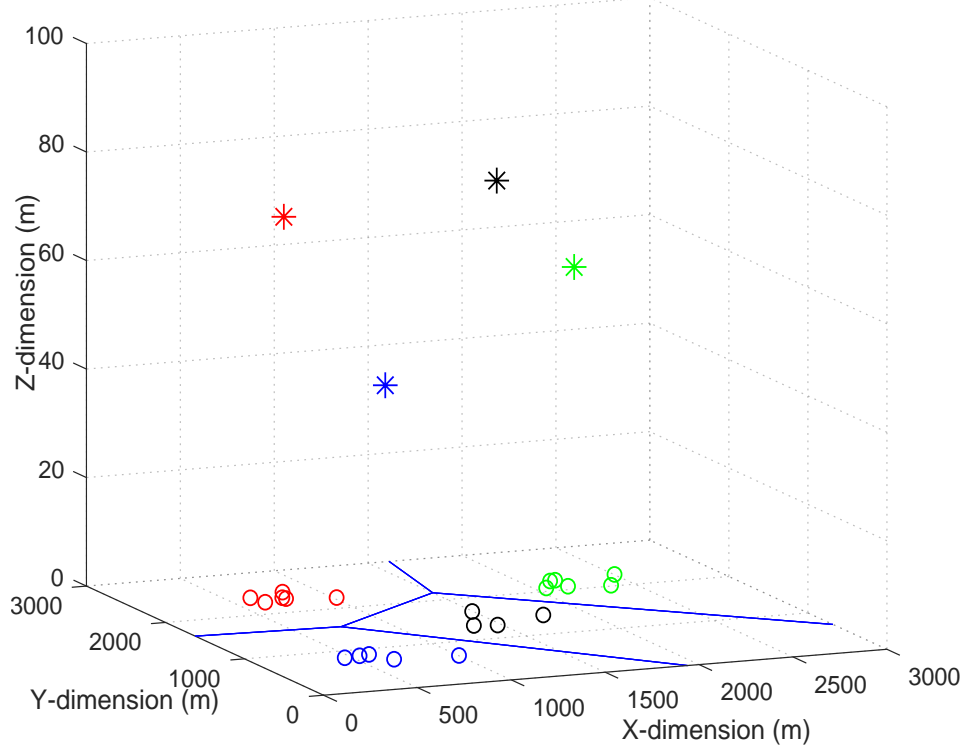

Fig. 3: The 3D multi-UAV placement and corresponding user scheduling.

for providing communication services might not be efficient when the received signal at users is corrupted by co-channel interference. In addition, flying at the higher altitudes can decrease the effects of co-channel interference by increasing the Euclidean distance between the UAVs and users. In order to enhance the performance of communication services, it is necessary to jointly optimize UAVs transmit powers and altitudes. Moreover, with respect to the fact that we maximize the minimum system throughout for all ground users, there might be other situations that can happen in practice. For example, if scheduled users to a UAV are more affected by co-channel interference compared to other users, it might be better that the UAV works in higher altitudes as it can produce more interference to others. It is obvious that we are not able to interpret all situations, but Algorithm 2 can make a balance between UAVs altitudes and transmit powers to mitigate the effects of co-channel interference for different user distributions.

In Fig. 3, we generate users following PCP in the target area. 4 parent points and their corresponding children are plotted. The minimum allowed distance between UAVs is set as $d_{\min }=1000 \mathrm{~m}$. Once users are scheduled by Algorithm 1. we implement Algorithm 2. The 3D deployment of UAVs is demonstrated in Fig. 3 The optimal UAVs transmit powers for the black, red, blue, and green UAV are $0.93,0.78,0.53$, $0.71 \mathrm{~W}$, respectively. Also, the optimal UAVs altitudes of the black, red, blue, and green UAV are 80.85, 70.02, 51.02, 58.15 $m$, respectively. It is observed that the black UAV that makes more co-channel interference than other UAVs works in the higher altitude to reduce its co-channel interference effects.

\section{B. System Sum Throughput}

Intuitively, it can be seen that the system throughput highly depends on user distributions. More precisely, when users are intrinsically clustered, the proposed method extracts the 


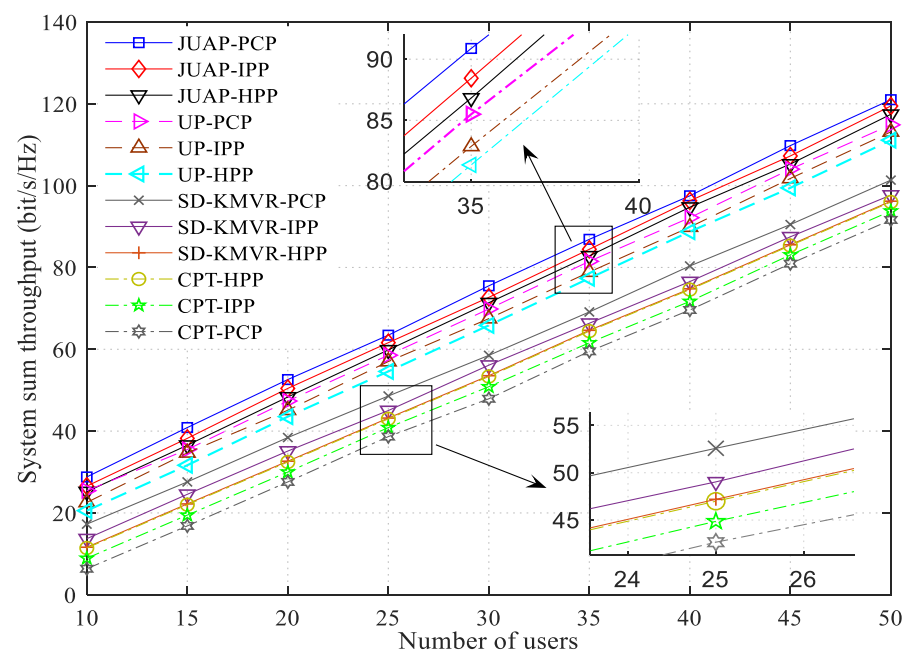

Fig. 4: Empirical system sum throughput (bits/s/Hz) versus the number of users for different user distributions, PCP, IPP, HPP in the target area with $s=3000$.

clustering properties and tries to deploy UAVs with the optimal altitudes and transmit powers in places where the density of users is high. On the other hand, when users are uniformly distributed in the target area, the Euclidean distance between UAVs and their scheduled users increases compared to the clustered users. Indeed, when the user points tend to have an uneven distribution, users enjoy better communication services as UAVs can be deployed in the places where are generally close to all of them. Therefore, it is expected that for the proposed approach, the system sum throughput for PCP is more than IPP and HPP. Since K-mean algorithm is used in SD-KMVR [15], similar to our approach, it is able to extract cluster properties of users. Therefore, we expect that the system sum throughput for PCP is more than other distributions. Conversely, it can be inferred that when UAVs are fixed in their locations, where happens in the CPT technique, the uniformly distributed users are more likely to be closed to UAVs. Hence, it is not surprising to see that the system sum throughput for HPP is more than IPP and PCP when CPT is employed to place UAVs.

In the same number of users, it is obvious that the number of UAVs to serve ground users for PCP is less than HPP and IPP because our approach does not deploy UAVs in places where users do not exist (see Fig. 5 (a)). Consequently, the effects of co-channel interference can considerably decrease in PCP case. Thus, we expect in the proposed approaches that are able to extract the clustering features of users, the system sum throughput for PCP is more than HPP and IPP. Another important point is that the proposed JUAP deploys UAVs with the optimal altitudes and transmit powers to mitigate the effects of co-channel interference. Therefore, it is expected such an optimal deployment and resource allocation lead to better performance in terms of system sum throughput compared to the benchmarks, SD-KMVR and CPT.
The above effects are illustrated in Fig. 4, which we compare the empirical system sum throughput of the proposed approach versus the number of users with the conventional benchmarks for different types of user distributions, PCP, IPP, HPP in the target area $3 \times 3 \mathrm{~km}^{2}$. Each point of the experiment was achieved over 50 trials. It can be observed that the system sum throughput of all methods increases monotonically with the number of users. Moreover, it is obvious that the performance of the approaches is affected by different types of distributions. Note that the performance of the conventional approaches is always less than the proposed deployment methods even for HPP. This is because of the fact that the proposed approaches deploy UAVs in places where the user's density is high as well as exploits the optimal values of UAV's altitudes and transmit powers. Indeed, the proposed deployment approach is not restricted to fixed locations, then, it can deploy UAVs according to the change of user distributions with the optimal UAVs altitudes and transmit powers. As a result, the effects of co-channel interference can be reduced significantly. Although SD-KMVR can find the cluster properties of users, UAVs altitudes and transmit powers are designed to maximize the coverage probabilities [15], not to mitigate co-channel interference. Hence, the performance of SD-KMVR is always less than our proposed approaches. Fig. 4 also demonstrates that when UAV's altitudes and transmit powers are jointly optimized, i.e., with the proposed JUAP, one can achieve a gain more than $67 \%$ in the system sum throughput compared to the benchmarks. The second approach for deploying multi-UAVs presented in this paper, i.e., UP, shows gains of up to $60 \%$ compared to the benchmarks as UAVs transmit powers are optimized to mitigate co-channel interference.

\section{Number of UAVs Required and Energy Efficiency}

In Fig. 5, we compare the number of UAVs required to serve ground users and the total transmit power for the proposed approach with the conventional benchmarks for different user distributions. Each point of the experiment was carried out over 50 trials. As explained in Section IV] the required number of UAVs depends only on Algorithm 1. Regarding this in Fig. 5 (a), we compare CPT with Algorithm 1. Note that the required number of UAVs for Algorithm 1 and SD-KMVR is almost the same because SD-KMVR uses the K-mean method which is a special case of the mean-shift technique employed in Algorithm [1 [26] (see [15, Fig. 11]).

In practice, users might gather around some specific spots in a target area such as rural areas or sports stadiums. It is not cost-effective to deploy UAVs in places where ground users do not exist out of the spots. Contrary to CPT that places UAVs in fixed locations, the proposed algorithm deploys UAVs in the locations where the user's density is high. These locations are the best places in terms of the average distance of users from UAVs. Thus, we expect that our technique reduces the required number of UAVs to serve ground users in any user distribution compared to CPT. To evaluate this, in Fig. 5 (a), we plot the number of UAVs versus the length of the target area for Algorithm 1 and CPT. This figure shows significant 


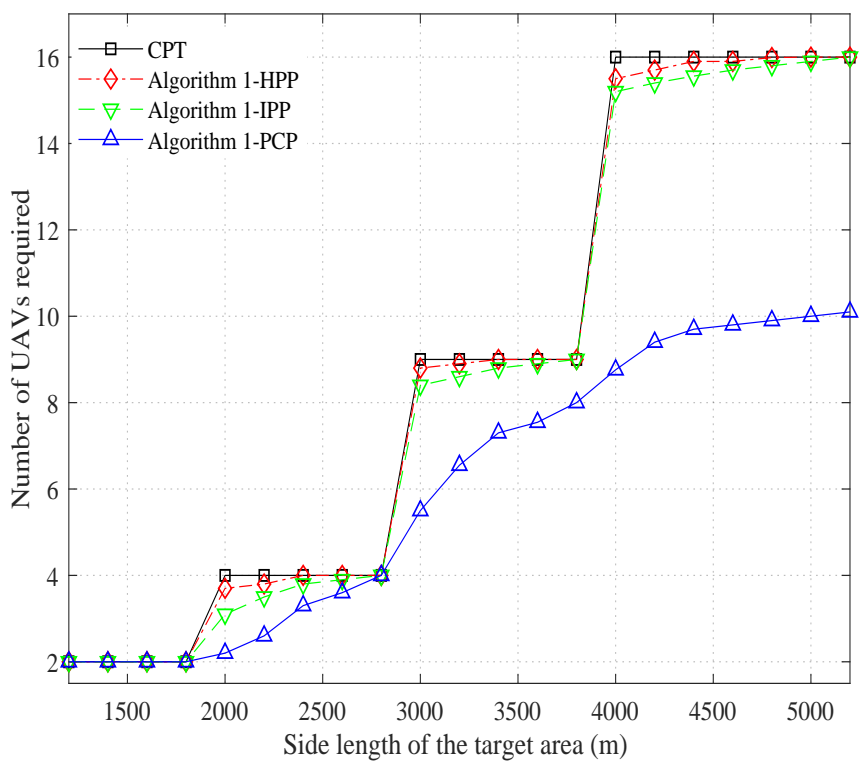

(a)

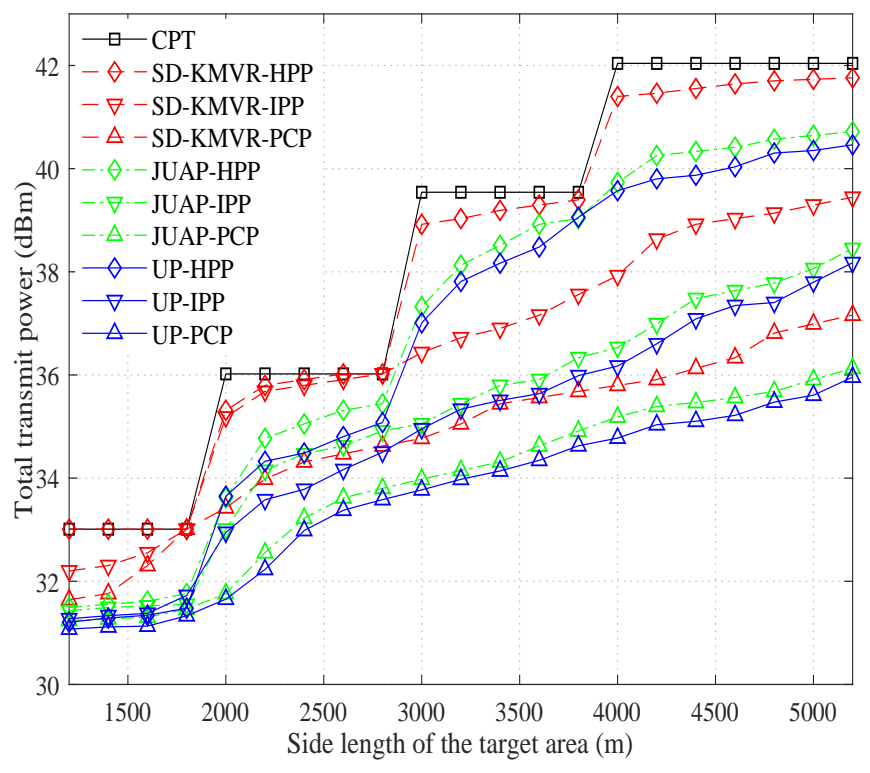

(b)

Fig. 5: The required number of UAVs and the total transmit power versus the size of the target area are plotted in Figs. 5 (a) and (b), respectively.



(a)

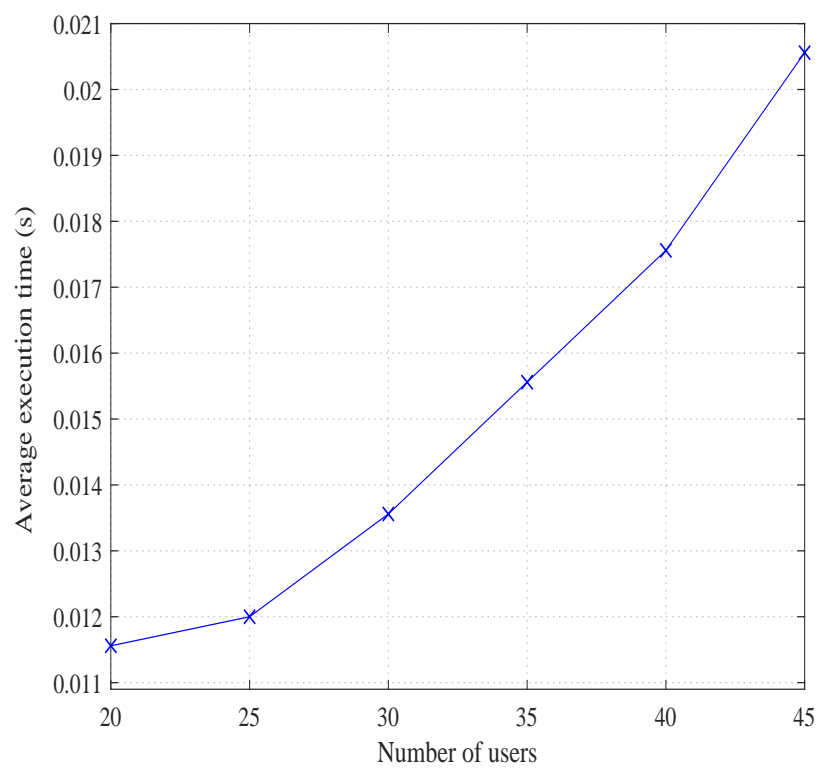

(b)

Fig. 6: The convergence behavior and computational complexity of Algorithm 1 are plotted in Figs. 6(a) and (b), respectively.

savings in the number of UAVs with a reduction of up to $6 \mathrm{UAVs}$ for the scenario evaluated compared to CPT. In particular, in Fig. 3, one can understand that in the area $[1000,3000] \times[0,1000] \mathrm{m}^{2}$ where there exist no users, the proposed approach does not allocate any UAVs to this area. Moreover, the gap between the required number of UAVs for Algorithm 1 and CPT significantly increases in the PCP case when the size of the target area increases. This is also because of the clustering features of users. However, when the users are uniformly distributed in the target area, HPP, the number of UAVs required to serve the ground users is almost equal to CPT that does not take into account the user distributions.
It is also observed that in IPP, the required number of UAVs is slightly less than CPT, which confirms the ability of the proposed approach to extract user classifications. Note that our proposed approach uses CPT to set $d_{\min }$ as explained in the first paragraph of Section $[\mathrm{IX}$ thus the number of UAVs to serve the ground users in our approach is upper bounded by CPT for different user distributions in any target area.

It is worth pointing out that in practice we might have a limited number of UAVs to serve the ground users. Regarding the fact that our approach automatically determines the number of UAVs, there might be a case in which the number of available UAVs is smaller than the result of Algorithm 1 1 . To 




(a)

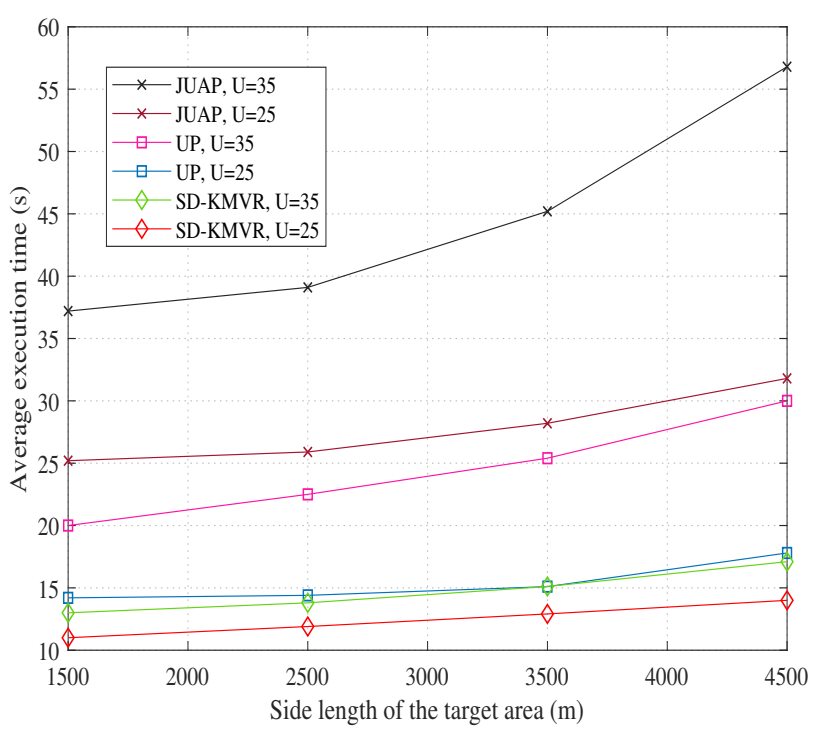

(b)

Fig. 7: The convergence behavior of Algorithm 2 is shown in Fig. 7(a). The computational complexity of JUAP in comparison with UP and SD-KMVR is drawn in Fig. 7 (b). Each point of the simulation shows the average execution time for the convergence of proposed approaches and implementing SD-KMVR over 50 trials.

overcome this practical issue, one can implement Algorithm 1 with different $d_{\min }$. As explained in Section IV] $\lambda$ can control the area around each mean-shift center. On the other hand, $\lambda \propto d_{\min }$. Therefore, we can implement Algorithm 1 for different amounts of $d_{\min }$ to obtain a user classification suitable for that number. Also, as proved in Section VIII, the computational cost of Algorithm 1 is low, thus this procedure can be efficiently implemented in practical scenarios.

Once the $x y$-Cartesian coordinates of UAVs determined using Algorithm 1. we jointly optimize UAV's altitudes and transmit powers via Algorithm 2 By setting $P_{\max }=1$ in the optimization problem in 9 , we ensure that the maximum power consumed by the system is upper bounded by CPT that all UAVs work with the maximum allowed transmit power. On the other hand, our proposed approach optimally allocates transmit powers to UAVs in order to maximize the minimum achievable system throughput. Thus, we expect that the total power consumed by the system in our proposed approach is less than the benchmarks. To investigate this, in Fig. 5 (b), we compare the total power consumed by the proposed approaches with the traditional methods for different types of user distributions. It can be observed that UP in the PCP serves as a lower bound for the total power consumed by the system because UAVs are deployed in the minimum allowed altitude. As expected, the power consumed by the system in CPT serves as an upper bound for all other techniques because UAVs always transmit data with the maximum allowed power. Also, our approach is superior to SD-KMVR because the proposed optimization in SD-KMVR is trying to optimize the radius of UAVs in order to maximize coverage region. Indeed, it is observed from this figure that by optimizing UAVs transmit powers, we can significantly reduce by up to $6 \mathrm{dBm}$ the total power consumed by the system. Besides extracting the clustering features of the ground users, which leads to reducing the required number of UAVs, optimizing UAVs transmit powers results in better performance in the term of the total power consumed by the system. Thus, our proposed approach outperforms the benchmarks.

It is observed from Figs. 4 and 5 (b) that it is possible to achieve better performance in terms of system sum throughput and total power consumed by the system by optimizing UAVs altitudes and transmit powers. This can ensure a longer operation compared to the conventional approaches. Note that regarding the fact that we maximize the minimum achievable system throughput for all ground users, communication services will be provided for all of them.

\section{Convergence and Computational Complexity of the De- ployment Approach}

As explained in previous sections, the proposed deployment approach can be divided into Algorithms 1 and 2 The cumulative distribution function (CDF) to investigate the convergence behavior of Algorithm 1 is plotted in Fig. 6 (a). The corresponding user distribution is demonstrated in Fig. 3 It can be observed that Algorithm 1 can converge in about 25 iterations. In Fig. 6 (b), we draw the average execution time versus the number of users to evaluate the computational complexity of Algorithm 1 . From Fig. 6 (b), it can be seen that Algorithm 1 can be implemented quickly. For each point of the experiment, users were uniformly generated in the target area over 50 trials. It is observed that the computational complexity increases as the number of users grows, confirming the theoretical results in 31 .

The convergence behavior of Algorithm 2 is investigated in Fig. 7 (a), which is shown the minimum system throughput 




Fig. 8: Fairness among ground users in terms of system throughput versus the number of users over 100 trials.

versus the number of iterations. This figure demonstrates that Algorithm 2 can converge in about 15 iterations for its associated user distributions plotted in Fig. 3. It is worth noting that all users access at least $2.8 \mathrm{bit} / \mathrm{s} / \mathrm{Hz}$ throughput. In order to evaluate the complexity costs of the placement approaches, we compare the average execution time versus the side length of the target area for different number of users for JUAP, UP and SD-KMVR in Fig. 77 (b) because as derived in Section VIII. the computational complexity of the proposed approaches is a function of both number of users and UAVs. Users were uniformly distributed in the target area. Each point of simulations shows the average execution time until convergence for the proposed approaches and implementing SD-KMVR over 50 trials. As discussed in Section VIII and observed in Fig. 7 (b), JUAP implementation takes more execution time than UP. Note that the computational complexity of CPT is low as the locations of UAVs are fixed, hence, we did not plot it. From Figs. 6 (b) and 7 (b), it is inferred that the complexity of Algorithm 11 can be ignored compared to the complexity of Algorithm 2 for the moderate number of users, which is consistent with the theoretical results derived in (33). Eventually, from Figs. 4 and 7 (b), one can understand that the superiority of JUAP in terms of system sum throughput compared to UP and other conventional approaches is obtained in cost of implementation complexity.

\section{E. Fairness Among Ground Users}

Intuitively, maximizing the minimum achievable system throughput for all ground users and deploying UAVs in locations where the average distance between users and UAVs is minimum provide fairness among users in terms of system throughput. However, there exists no such a consideration in the conventional approaches, they are therefore tailored towards the stronger users, thus reducing fairness in the system throughput. To investigate this metric, we first define a Jain's index [32] which is given by

$$
f_{j}:=\frac{\left(\sum_{u=1}^{U} R_{u}\right)^{2}}{U \sum_{u=1}^{U} R_{u}^{2}},
$$

that evaluates fairness among ground users in terms of system throughput. Accordingly, a high degree of fairness results in $f_{j}$ values close to one and the lowest is $1 / U$. For this experiment, we consider a target area $2 \times 2 \mathrm{~km}^{2}$ and set $d_{\text {min }}=1000 \mathrm{~m}$. Then, we compare $f_{j}$ for the proposed UP, JUAP with the benchmarks for different numbers of users in different types of user distribution. In Fig. 8, we plot $f_{j}$ versus the number of users that shows our proposed approaches outperform up to $17 \%$ the benchmarks in terms of fairness. It can be also seen that $f_{j}$ slightly decreases by increasing the number of users which is due to increasing the average distance of ground users from the UAVs. Moreover, when users form clusters, PCP and IPP, the proposed approaches and SD-KMVR obtain better performance compared to the uniformly distributed case, HPP. This is because of extracting the user's classification feature of proposed approaches and SD-KMVR. However, in CPT, due to the fact that UAVs are placed at fixed locations, better performance in terms of fairness can be achieved in HPP.

\section{Conclusion and Future Research Directions}

In this paper, we proposed a 3D deployment approach to provide communication services to all ground users in multiUAV communication frameworks. The resulting problem was a combination of the integer and non-convex constraints with the system throughput maximization as its objective, hence difficult to solve. We first proposed a low complexity algorithm to schedule users to UAVs and find $x y$-Cartesian coordinates of UAVs. The suboptimal UAVs altitudes and transmit powers are separately obtained by the successive convex approximation. The iterative algorithm using the block coordinate descent was developed to jointly optimize UAVs altitudes and transmit powers. The simulation results confirmed that the proposed approach is guaranteed to converge and unveiled that the this method can be applied to different user distributions and achieves a significant enhancement in system sum throughput compared to the conventional benchmarks with less power consumed in the cost of more complexity.

There exist several interesting future directions that can be explored. One may want to relax the integer constraints in $9 \mathrm{~b}$ by $0 \leq \alpha_{u, m} \leq 1$. Also, the non-convex constraint in $9 \mathrm{e}$ can be implemented by its lower bound obtained by its first-order Taylor expansion. It is beneficial if one applies the mentioned techniques and compares the system sum throughput with our deployment approach. Moreover, it would be nice if one could extend the approach [21] to the multi-UAV scenario with cochannel interference and sees if there exists a similar trade-off between communication services and energy harvested from the sun. 


\section{ACKNOWLEDGMENT}

Iman Valiulahi is thankful to Qingqing Wu for helpful email discussions related to the implementation of equation (28) of his work in [7].

\section{APPENDIX A \\ PROOF OF THEOREM 1}

Since the optimal objective value of problem (24) is obtained for $\boldsymbol{p}^{t}$ and step 3 of Algorithm 2, we have

$$
\psi\left(\boldsymbol{z}^{t}, \boldsymbol{p}^{t}\right) \leq \psi\left(\boldsymbol{z}^{t+1}, \boldsymbol{p}^{t}\right) .
$$

Also, for given $z^{t+1}$ and step 4 of Algorithm 2, we have

$$
\begin{aligned}
\psi\left(\boldsymbol{z}^{t+1}, \boldsymbol{p}^{t}\right) & =\psi_{\boldsymbol{p}}\left(\boldsymbol{z}^{t+1}, \boldsymbol{p}^{t}\right) \\
& \leq \psi_{\boldsymbol{p}}\left(\boldsymbol{z}^{t+1}, \boldsymbol{p}^{t+1}\right) \\
& \leq \psi\left(\boldsymbol{z}^{t+1}, \boldsymbol{p}^{t+1}\right),
\end{aligned}
$$

where the equality comes from the fact that the first-order Taylor expansion used in 28 is tight at the local point. Indeed, the objective value of problem $\sqrt{30}$ at $\boldsymbol{p}^{t}$ is equal to the objective value of problem 25). The first inequality holds because the objective value of problem (30) is optimally obtained in step 4 of Algorithm 2 The second inequality stems from the fact that the objective value of problem (30) serves as a lower bound for the objective value of its main problem 25 . Thus, the objective value of problem (25) is non-decreasing in each iteration though we solve it approximately.

From (35) and 36, one can write

$$
\psi\left(\boldsymbol{z}^{t}, \boldsymbol{p}^{t}\right) \leq \psi\left(\boldsymbol{z}^{t+1}, \boldsymbol{p}^{t+1}\right) .
$$

This inequality implies the convergence of proposed Algorithm 2 because the objective value of problem (13) is upper bounded by a finite value. This concludes the proof.

\section{REFERENCES}

[1] J. G. Andrews, S. Buzzi, W. Choi, S. V. Hanly, A. Lozano, A. C. Soong, and J. C. Zhang, "What will 5g be?", IEEE Journal on selected areas in communications, vol. 32, no. 6, pp. 1065-1082, 2014.

[2] V. W. Wong, Key technologies for $5 G$ wireless systems. Cambridge university press, 2017.

[3] Y. Zeng, R. Zhang, and T. J. Lim, "Wireless communications with unmanned aerial vehicles: Opportunities and challenges," IEEE Communications Magazine, vol. 54, no. 5, pp. 36-42, 2016.

[4] Y. Zeng, R. Zhang, and T. J. Lim, "Throughput maximization for uavenabled mobile relaying systems," IEEE Transactions on Communications, vol. 64, no. 12, pp. 4983-4996, 2016.

[5] Y. Zeng and R. Zhang, "Energy-efficient uav communication with trajectory optimization," IEEE Transactions on Wireless Communications, vol. 16, no. 6, pp. 3747-3760, 2017.

[6] Y. Zeng, X. Xu, and R. Zhang, "Trajectory design for completion time minimization in uav-enabled multicasting," IEEE Transactions on Wireless Communications, vol. 17, no. 4, pp. 2233-2246, 2018.

[7] Q. Wu, Y. Zeng, and R. Zhang, "Joint trajectory and communication design for multi-uav enabled wireless networks," IEEE Transactions on Wireless Communications, vol. 17, no. 3, pp. 2109-2121, 2018.

[8] Y. Sun, D. Xu, D. W. K. Ng, L. Dai, and R. Schober, "Optimal 3d-trajectory design and resource allocation for solar-powered uav communication systems," IEEE Transactions on Communications, 2019.

[9] M. Mozaffari, W. Saad, M. Bennis, and M. Debbah, "Unmanned aerial vehicle with underlaid device-to-device communications: Performance and tradeoffs," IEEE Transactions on Wireless Communications, vol. 15, no. 6, pp. 3949-3963, 2016.

[10] M. Mozaffari, W. Saad, M. Bennis, and M. Debbah, "Efficient deployment of multiple unmanned aerial vehicles for optimal wireless coverage," IEEE Communications Letters, vol. 20, no. 8, pp. 1647-1650, 2016.
[11] A. Al-Hourani, S. Kandeepan, and S. Lardner, "Optimal lap altitude for maximum coverage," IEEE Wireless Communications Letters, vol. 3 , no. 6, pp. 569-572, 2014.

[12] J. Lyu, Y. Zeng, R. Zhang, and T. J. Lim, "Placement optimization of uav-mounted mobile base stations," IEEE Communications Letters, vol. 21, no. 3, pp. 604-607, 2016.

[13] M. Alzenad, A. El-Keyi, F. Lagum, and H. Yanikomeroglu, "3-d placement of an unmanned aerial vehicle base station (uav-bs) for energyefficient maximal coverage," IEEE Wireless Communications Letters, vol. 6, no. 4, pp. 434-437, 2017.

[14] M. Alzenad, A. El-Keyi, and H. Yanikomeroglu, "3-d placement of an unmanned aerial vehicle base station for maximum coverage of users with different qos requirements," IEEE Wireless Communications Letters, vol. 7, no. 1, pp. 38-41, 2017.

[15] J. Sun and C. Masouros, "Deployment strategies of multiple aerial bss for user coverage and power efficiency maximization," IEEE Transactions on Communications, vol. 67, no. 4, pp. 2981-2994, 2018.

[16] A. Merwaday and I. Guvenc, "Uav assisted heterogeneous networks for public safety communications," in 2015 IEEE wireless communications and networking conference workshops (WCNCW), pp. 329-334, IEEE, 2015.

[17] S.-Y. Lien, K.-C. Chen, and Y. Lin, "Toward ubiquitous massive accesses in 3gpp machine-to-machine communications," IEEE Communications Magazine, vol. 49, no. 4, pp. 66-74, 2011.

[18] M. Mozaffari, W. Saad, M. Bennis, and M. Debbah, "Mobile unmanned aerial vehicles (uavs) for energy-efficient internet of things communications," IEEE Transactions on Wireless Communications, vol. 16, no. 11, pp. 7574-7589, 2017.

[19] N. Dlodlo and J. Kalezhi, "The internet of things in agriculture for sustainable rural development," in 2015 international conference on emerging trends in networks and computer communications (ETNCC), pp. 13-18, IEEE, 2015.

[20] K. Kanistras, G. Martins, M. J. Rutherford, and K. P. Valavanis, "A survey of unmanned aerial vehicles (uavs) for traffic monitoring," in 2013 International Conference on Unmanned Aircraft Systems (ICUAS), pp. 221-234, IEEE, 2013.

[21] Y. Sun, D. W. K. Ng, D. Xu, L. Dai, and R. Schober, "Resource allocation for solar powered uav communication systems," in 2018 IEEE 19th International Workshop on Signal Processing Advances in Wireless Communications (SPAWC), pp. 1-5, IEEE, 2018.

[22] E. Kalantari, H. Yanikomeroglu, and A. Yongacoglu, "On the number and $3 \mathrm{~d}$ placement of drone base stations in wireless cellular networks," in 2016 IEEE 84th Vehicular Technology Conference (VTC-Fall), pp. 16, IEEE, 2016.

[23] M. Hong, M. Razaviyayn, Z.-Q. Luo, and J.-S. Pang, "A unified algorithmic framework for block-structured optimization involving big data: With applications in machine learning and signal processing," IEEE Signal Processing Magazine, vol. 33, no. 1, pp. 57-77, 2015.

[24] A. Al-Hourani, S. Kandeepan, and A. Jamalipour, "Modeling air-toground path loss for low altitude platforms in urban environments," in 2014 IEEE global communications conference, pp. 2898-2904, IEEE, 2014.

[25] Q. Feng, J. McGeehan, E. K. Tameh, and A. R. Nix, "Path loss models for air-to-ground radio channels in urban environments," in 2006 IEEE 63rd vehicular technology conference, vol. 6, pp. 2901-2905, IEEE, 2006.

[26] D. Comaniciu and P. Meer, "Mean shift analysis and applications," in Proceedings of the Seventh IEEE International Conference on Computer Vision, vol. 2, pp. 1197-1203, IEEE, 1999.

[27] S. Boyd and L. Vandenberghe, Convex optimization. Cambridge university press, 2004.

[28] M. Grant, S. Boyd, and Y. Ye, "cvx users' guide," online: http://www. stanford. edu/ boyd/software. html, 2009.

[29] M. Grant, S. Boyd, and Y. Ye, "Cvx: Matlab software for disciplined convex programming," 2008.

[30] J. Illian, A. Penttinen, H. Stoyan, and D. Stoyan, Statistical analysis and modelling of spatial point patterns, vol. 70. John Wiley \& Sons, 2008.

[31] A. Baddeley, I. Bárány, and R. Schneider, "Spatial point processes and their applications," Stochastic Geometry: Lectures Given at the CIME Summer School Held in Martina Franca, Italy, September 13-18, 2004, pp. 1-75, 2007.

[32] R. K. Jain, D.-M. W. Chiu, W. R. Hawe, et al., "A quantitative measure of fairness and discrimination," Eastern Research Laboratory, Digital Equipment Corporation, Hudson, MA, 1984. 